\title{
La syntaxe de la forme II de l'arabe classique
}

\section{Nora Arbaoui}

\section{OpenEdition}

Journals

Édition électronique

URL : http://journals.openedition.org/rlv/1838

DOI : $10.4000 /$ rlv. 1838

ISSN : 1958-9239

\section{Éditeur}

Presses universitaires de Vincennes

\section{Édition imprimée}

Date de publication : 1 décembre 2010

Pagination : 69-94

ISBN : 978-2-84292-264-1

ISSN : 0986-6124

\section{Référence électronique}

Nora Arbaoui, "La syntaxe de la forme II de l'arabe classique », Recherches linguistiques de Vincennes [En ligne], 39 | 2010, mis en ligne le 01 décembre 2014, consulté le 19 avril 2019. URL : http:// journals.openedition.org/rlv/1838; DOI : 10.4000/rlv.1838 
Recherches Linguistiques de Vincennes 39 - 2010 - p. 69-94

\section{Nora ARBAOUI \\ Université Paris 7}

\section{LA SYNTAXE DE LA FORME II DE L'ARABE CLASSIQUE}

\section{RÉSUMÉ}

La forme II de l'arabe classique gémine la deuxième consonne de la racine et dénote plusieurs significations: le causatif, l'intensif, l'estimatif et le dénominatif. L'analyse morphophonologique proposée par Guerssel \& Lowenstamm (1990) montre l'existence d'une position (CV) dérivationnelle dans un gabarit de forme: $\mathrm{CV}(\mathrm{CV}) \mathrm{CVCV}$, responsable de la réalisation de la géminée. Cependant, dans une telle analyse, les diverses interprétations qu'exprime cette forme ne trouvent pas d'explication.

Dans cet article, nous proposons une analyse syntaxique de la forme II de l'arabe classique, laquelle permet d'apporter une réponse quant à l'origine de ce (CV) dérivationnel et des positions CV du gabarit de base. De même, elle permet d'expliquer les différentes interprétations sémantiques que peut dénoter cette forme. La forme II est analysée de la même manière qu'un verbe causatif en français: elle renferme une structure englobant un VP, un vP en plus d'une projection $\sqrt{ } \mathrm{P}$. La racine est présentée non seulement comme étant une suite de trois consonnes mais aussi comme constituant un domaine où elle projette ses propres arguments.

Cet article montre l'intérêt de la considération de la racine et illustre la solution que peut apporter la syntaxe à des problèmes considérés comme étant d'ordre morphophonologique.

\section{MotS-CLÉS}

Interface phonologie-syntaxe, arabe classique, forme II, structure de la racine, intensif, causatif, gabarit. 
Dans cet article, nous nous proposons de déterminer la structure syntaxique de la forme II, de révéler l'origine de la position CV dérivationnelle qui produit la géminée ainsi que celle de toutes les positions CV du gabarit proposé par Guerssel \& Lowenstamm $(1990)^{1}$. Nous dévoilerons une nouvelle organisation de la racine: une racine qui projette ses propres arguments et dont la structure est déterminante quant au devenir de la forme verbale.

Tout au long de ce travail sera établie l'importance primordiale de la considération de la racine - par opposition à la notion de radical ou de stem dans l'analyse des formes verbales de l'arabe classique, plus particulièrement la forme II. Nous avons choisi de traiter cette forme pour sa particularité morphologique mais aussi sémantique: une forme qui gémine la deuxième consonne et qui indique un sens causatif ou un sens intensif. La question de savoir quel est le lien entre le causatif et l'intensif et comment une seule forme peut indiquer à la fois ces deux notions, se pose d'emblée. De surcroit, la forme II exprime un troisième sens qu'on omet souvent de signaler à savoir le sens estimatif. Elle peut avoir également un emploi dénominatif.

Guerssel \& Lowenstamm (1990) proposent une analyse morphophonologique de la forme II qui met en œuvre un site dérivationnel auquel est associée la deuxième consonne de la racine et qui est responsable de sa gémination:

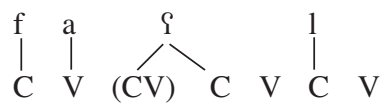

Avec cette proposition, on sait comment est obtenue une forme II mais on ne sait pas encore, par exemple, pourquoi elle indique le causatif et l'intensif plutôt que le réflexif ou le réciproque.

En réalité, on ne peut pas comprendre comment la forme II peut exprimer à la fois le causatif et l'intensif, sans oublier l'estimatif, tant qu'on ne sait pas ce qu'est réellement une forme II. Il faudra se demander, d'abord, pourquoi et comment une forme qui gémine la deuxième consonne de la racine indique le causatif. Pour cela, il faut aller au-delà des frontières de la phonologie et de la morphologie et chercher la réponse dans la syntaxe.

Dans cette analyse, nous présenterons le domaine VP sous une autre apparence et nous ferons appel à la projection vP. Nous montrerons que la racine n'est pas un élément terminal de $\mathrm{V}$ et qu'au contraire, elle a sa propre projection. Cette propriété est essentielle quant à l'explication des différentes interprétations sémantiques que peut dénoter la forme II. Nous établirons l'existence de la racine en tant que suite exclusivement consonantique, condition

1. Ce gabarit est mis en œuvre dans plusieurs travaux portant sur la morphologie de diverses langues afro-asiatiques (voir Benjaballah,2007, Lahrouchi \& Ségéral, 2009 , etc.). 
nécessaire, voire indispensable, pour l'explication de la morphologie de la forme II. Cette vision de la racine permet de réaliser une analyse syntaxique pour les neuf autres formes verbales de l'arabe classique et permet d'élucider des problèmes jusqu'alors non résolus.

Cette étude s'inspire de ce qui est l'essence même de la Morphologie Distribuée, à savoir la possibilité d'analyser des mots en syntaxe. Cependant, elle s'inscrit directement dans le cadre général de la syntaxe générative: le lecteur remarquera, par exemple, que la projection vP est utilisée ici non pas pour assigner la catégorie verbe à la racine comme soutenu en Morphologie Distribuée, mais pour introduire un deuxième niveau verbal, utilisé en syntaxe pour rendre compte, entre autres, du causatif. Des éléments morphophonologiques sont directement insérés dans des arbres syntaxiques afin de rendre visible l'interface syntaxe/phonologie à chaque étape de la construction d'une forme.

\section{La forme II causative}

Il s'agit de l'emploi le plus connu pour la forme II. C'est en étudiant la forme causative que l'on établira la structure syntaxique d'une forme II fa९ৎal, après quoi elle servira de base de comparaison pour les autres usages non causatifs de la forme II.

Soit les exemples suivants d'une forme II causative:

(2) Sallama kariim-un Saliyy-an as-sibaahat-a

a enseigné karim-nominatif @ali-accusatif la natation-accusatif

'karim a enseigné la natation à Sali'

(3) farraћa al-?ab-u at-tifl-a

a rendu content le père-nominatif l'enfant-accusatif

'le père a rendu l'enfant content'

À la forme I correspondante on a:

(4) Salima Saliyy-un al-haqiiqat-a

a su Cali-nominatif la vérité-accusatif

'Sali a su la vérité'

(5) farifa at-tiflu

est content l'enfant-nominatif

'l'enfant est content'

Les exemples avec les formes II laissent apparaître un argument supplémentaire par rapport à ceux des formes I correspondantes. De surcroit, les formes II citées ci-dessus, réalisent un prédicat supplémentaire par rapport aux formes I correspondantes: 
(6) Salima $\rightarrow$ Sallama

$$
\text { [X sait] [Y fait X sait] }
$$

(7) fariћa $\rightarrow$ farraћa

[X est content] [Y fait $\mathrm{X}$ est content]

\subsection{Structure syntaxique de la forme II causative}

Les différents exemples cités ci-dessus montrent que la forme II apporte un prédicat du type «faire/ rendre» et ajoute un argument externe (un agent causateur). Ces deux propriétés font la structure d'une forme II causative.

Reprenons un des exemples cités plus haut:

(8) Sallama kariim-un Saliyy-an as-sibaahat-a

a enseigné karim-nominatif Sali-accusatif la natation-accusatif

karim a enseigné la natation à Yali

La forme II Yallama est à traduire par 'enseigner' et se laisse décomposer en [faire + savoir]. On peut reconstituer la proposition comme suit: [Karim fait [Sali sait]]. Lorsqu'on passe d'une forme simple Salima à une forme II Sallama, on indique que le Sujet, argument ajouté par la forme II, est l'Agent de «faire » et que l'argument Objet reste le Thème de «savoir». Autrement dit, on garde la proposition [Sali sait] et on lui ajoute une structure de causatif, de façon à indiquer que cet événement à une cause extérieure: [Karim fait].

De la sorte, l'argument ajouté par la structure du causatif n'a pas un lien direct avec l'événement «savoir». Seul l'objet de Sallamaqui est le thème de Salima, est concerné par cet événement. Cette hypothèse est appuyée par le fait qu'en arabe classique on dit:

(9) Sallam-tu-hu wa lam ya-taiallam

J'ai enseigné (à) lui et il n'a pas appris

'Je lui ai enseigné et il n'a pas appris'

Cela indique que l'agent de «faire»n'est pas concerné par «savoir». Il fait en sorte que l'objet «sache» mais ce dernier peut bien avoir acquis le savoir comme il peut bien ne pas l'avoir acquis.

De ce fait, la forme II se contente d'ajouter un verbe et un argument à l'expression de base sans changer le rôle thématique de l'argument de celle-ci. Cette propriété est primordiale dans la construction d'une forme II car c'est ce qui déterminera le type de mouvements que cette forme exécutera et c'est ce qui va la distinguer d'une autre forme verbale causative dans le système, à savoir la forme IV.

La forme II contient un verbe et un argument supplémentaire par rapport à la forme de base. De ce fait, elle inclut une projection supplémentaire par rapport à la forme I. Il s'ensuit que sa structure syntaxique englobe celle 
d'une forme I à laquelle est ajouté un niveau syntaxique supplémentaire. La partie qui sera partagée par ces deux formes est celle qui inclut la racine et le domaine VP.

\subsubsection{La racine}

Le débat entre partisans et opposants à l'idée qu'une racine contient des éléments de catégorie syntaxique reste à l'heure actuelle ouvert. Cependant, beaucoup s'accordent à dire qu'une racine contient un nombre de traits sémantiques tels que «objet», «qualité», «action», «état», etc.

Nous proposons de voir la racine sous un autre angle. Nous présumons que les racines qui contiennent des traits tels que «action», «état» contiennent aussi les participants à l'événement dénoté par la racine. Ce contenu sémantique se reflète dans la structure de la racine, voire de $\sqrt{ } \mathrm{P}$. En effet, selon le nombre d'arguments associés à une racine et selon le rôle thématique de chacun d'eux, l'architecture de $\sqrt{ } \mathrm{P}$ sera différente. Trois structures sont dénombrées.

Une racine comme $\sqrt{k t b}$ dont la matrice est:

$$
\left[\begin{array}{l}
\sqrt{ } \mathbf{k t b} \\
\text { ÉCRIRE } \\
\text { Action } \\
\text { Agent } \\
\text { But }
\end{array}\right]
$$

aura la structure suivante:

(11)

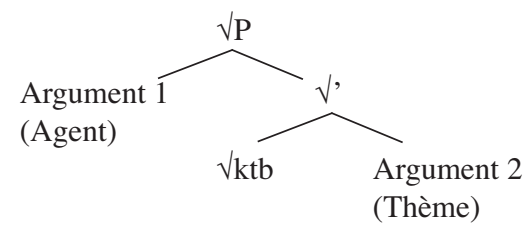

L'argument Agent prend la position Spécifieur et l'argument But prend la position Complément. Les racines qui ont deux arguments auront toutes cette même structure.

Une racine telle que $\sqrt{ } x r_{3}$ dont la matrice est:

$$
\left[\begin{array}{l}
\sqrt{ } \mathbf{x r 3} \\
\text { SORTIR } \\
\text { Action } \\
\text { Thème }
\end{array}\right]
$$

aura la structure suivante: 
(13)

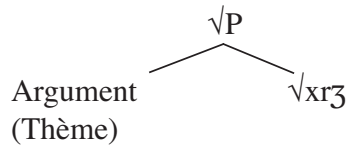

Le seul argument que comporte la racine a le rôle thématique Thème et occupe donc la position Spécifieur de $\sqrt{ } \mathrm{P}$. Ce sera le cas pour toutes les racines qui ont un seul argument dont le rôle thématique est Agent ou Thème. Ce genre de racine, une fois sélectionné par VP, donne à la forme I des verbes intransitifs ou inaccusatifs tels que «sortir» mais aussi «tomber». L'argument Agent ou Thème occupera, une fois $\sqrt{ } \mathrm{P}$ sélectionné ultimement par $\mathrm{VP}$, la position Spécifieur de VP et aura, par la suite, la fonction Sujet.

Une racine telle que $\sqrt{k s r}$ dont la matrice est:

$\left[\begin{array}{l}\sqrt{ } \mathbf{k s r} \\ \text { CASSER } \\ \text { Expérience } \\ \text { Patient }\end{array}\right]$

aura la structure suivante:

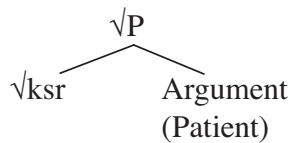

Le seul argument que détient cette racine a le rôle thématique Patient et occupe donc la position Complément de $\sqrt{ } \mathrm{P}$. Ce sera le cas pour toutes les racines dont le seul argument a le rôle thématique Patient. Cet argument, une fois $\sqrt{ } \mathrm{P}$ sélectionnée par VP, gardera sa position de Complément et aura la fonction d'Objet direct. Il ne prendra pas la position Spécifieur de VP et ne sera pas le Sujet dans une forme I kasar: *kasar al-ka?s ('a cassé le verre'). Un argument agent sera apporté par les projections sélectionnant cette racine de manière à la doter d'un sujet: Sali kasar al-ka?s. Il est intéressant de signaler que ce genre de racines est le seul qui peut avoir une forme VII n-faSal; ce qui justifie qu'on les distingue structurellement des autres racines à un seul argument du type $V_{x} r z$. De surcroit, ces racines lorsqu'elles sont à la forme II, indiquent un sens intensif au lieu d'un sens causatif, ce qui permet de les distinguer d'une racine comme $\sqrt{ } k t b$.

Les racines projetant des arguments sont prédestinées à être sélectionnées par VP qui assure une fonction syntaxique à ces derniers (Sujet, Objet). Cela n'empêche pas les racines qui n'ont pas d'arguments et qui formeront essentiellement des noms d'être également sélectionnées par VP (ce dernier peut apporter un argument et garantit donc l'aboutissement d'une prédication). 


\subsubsection{Le domaine VP}

Une fois qu'on a admis que les propriétés sémantiques que l'on attribuait aux verbes sont en réalité contenues dans la racine, il faudra se prononcer sur ce qu'on appelle «la grille thématique du verbe».

Certes, le domaine VP prévoit des positions pour disposer les différents arguments du verbe, mais il s'agit là de positions syntaxiques que doivent occuper des N. Il s'agit seulement de la projection syntaxique de la grille thématique. Aucun des constituants du domaine VP ne comporte les informations lexicales responsables de la détermination des rôles thêta. Ce qui est lexical dans un verbe, c'est la racine.

Avec l'hypothèse qu'une racine vient avec ses arguments, la grille thématique qu'on attribuait au verbe devient l'affaire de la racine et la projection VP se voit ôter ses constituants. En effet, la racine ainsi que les arguments prennent place non pas dans la projection VP mais dans la projection $\sqrt{ } \mathrm{P}$.

Privé de sa propriété d'être une tête lexicale, $\mathrm{V}$ ne peut que devenir une tête fonctionnelle. Certes, la racine organise ses propres arguments en Spécifieur et Complément de $\sqrt{\mathrm{P}}$ mais cette répartition est basée sur le rôle thématique de chacun. Ce n'est que lorsque la racine est sélectionnée par VP qu'elle reçoit ainsi que ces arguments une fonction syntaxique.

En montant dans la position $\mathrm{V}$, la racine acquiert le trait syntaxique [+verbe]. L'argument de la racine qui occupe la position Spécifieur de $\sqrt{ } \mathrm{P}$ se déplace dans la position Spécifieur de VP, ce qui lui permettra par la suite d'avoir le cas nominatif et la fonction Sujet. L'argument qui occupe la position Complément de $\sqrt{ } \mathrm{P}$ reste dans sa position de Complément par rapport à $\mathrm{V}$, ce qui lui vaut le cas accusatif et la fonction Objet direct:

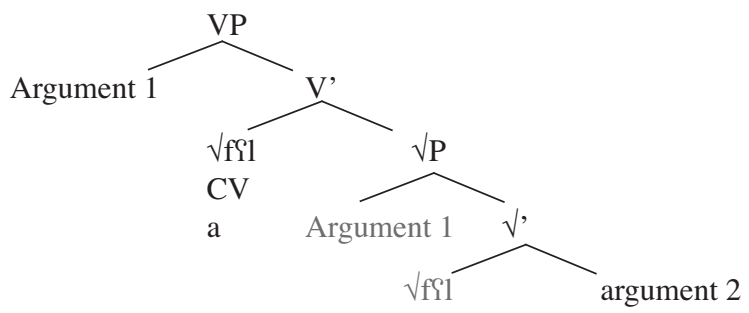

En tant que tête fonctionnelle, $\mathrm{V}$ disposera d'une position $\mathrm{CV}^{2}$. Et si l'on examine les dix formes verbales de l'arabe classique, on constate qu'elles réalisent toutes une voyelle $a$ en $\mathrm{V}_{1}$. Cette voyelle a été considérée tantôt

2. Dans son étude de l'afar, Rucart (2006) avait proposé que les têtes fonctionnelles contiennent une position CV. Nous proposons la même chose pour l'arabe classique où les têtes fonctionnelles sont souvent limitées à un $\mathrm{CV}$ et où plusieurs opérations morphologiques peuvent survenir au niveau et en considération d'un CV. 
comme une voyelle du perfectif tantôt comme une voyelle de l'actif et ce, parce qu'elle servait de voyelle de départ pour la dérivation de l'imperfectif et du passif (voir Guerssel \& Lowenstamm, 1996).

En effet, chacun du passif et de l'imperfectif contient une voyelle obtenue par apophonie à partir de la voyelle $a$. À signaler que certaines formes de l'imperfectif ne répondent pas à cette généralisation et gardent la voyelle $a$ intacte (c'est le cas, par exemple, de la forme I imperfective ya-ktub et de la forme VII imperfective ya-nkatib) tandis que toutes les formes du passif changent le timbre vocalique de cette voyelle et réalisent un $u$. Étant donné que la voyelle $a$ apparaît dans toutes les formes verbales mais aussi dans toutes les formes déverbales (formes participiales actives et passives et dans le masdar), nous considérons qu'elle est dans $\mathrm{V}$. De la sorte, la tête $\mathrm{V}$ contient une position $\mathrm{CV}$ et une voyelle $a$.

L'élément V agence les arguments de la racine en Sujet et Objet en respectant l'ordre que leur donne la racine. Lorsqu'on passe à la voix passive, s'opère un changement concernant la structuration des arguments qui voient leur fonction syntaxique bouleversée : l'argument Patient devient Sujet. Le fait que le changement de voix implique directement le changement de la position syntaxique des arguments indique que la structuration initiale en Sujet et Objet qui s'effectue en VP dépend, en réalité, de la voix active.

Ainsi, en dehors du fait de donner à la racine la catégorie syntaxique Verbe, on attribue à $V$ une fonction que l'on reconnaît habituellement à la voix active. La similitude entre ce que fait $\mathrm{V}$ et le rôle que l'on attribue à la voix active peut être expliquée en postulant tout simplement que la voix active est contenue dans V. La voix active n'est pas réalisée dans une projection VoixP mais simplement contenue dans $\mathrm{V}$, et c'est elle qui structure les arguments de la racine.

La structure telle qu'établie en (16) correspond à celle d'une forme I et sera présente dans chacune des dix formes verbales de l'arabe classique. L'élément $\mathrm{V}$ procure la première position $\mathrm{CV}$ du gabarit de base (CVCVCV) élaboré par Guerssel \& Lowenstamm. Dans une forme I, cette position sera associée à la première consonne de la racine:

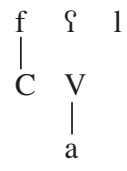

La racine n'apporte que les trois consonnes et c'est la structure de la forme verbale qui apporte les positions $\mathrm{CV}$ qui constituent un gabarit. Chaque consonne de la racine sera associée à une position CV. Par ailleurs, chaque tête fonctionnelle renfermera une position CV. 
La première consonne de la racine s'associe à la position CV apportée par V. Quant aux deux autres positions CV du gabarit auxquelles seront associées la deuxième et la troisième consonne de la racine, elles proviennent des têtes fonctionnelles que renferme la structure de la forme verbale. En effet, un verbe contient, en plus de $\sqrt{\mathrm{P}}$ et de VP, l'Aspect et les marques de Personne, de Genre et de Nombre. De la sorte, la deuxième position CV constituant le gabarit de base sera apportée par la tête fonctionnelle qui sélectionne VP à savoir AspectP et la troisième position $\mathrm{CV}$, quant à elle, sera apportée par AgreementP:

(18)

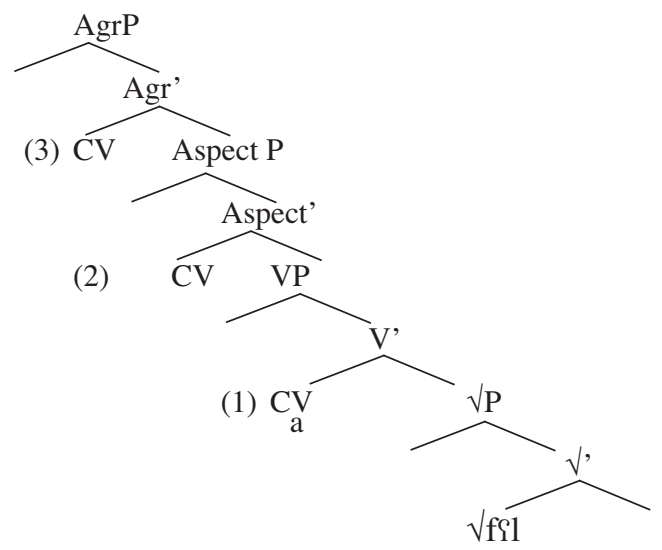

Étant donné que le perfectif n'a pas une marque consonantique, la position $\mathrm{C}$ du $\mathrm{CV}$ reste libre et peut donc être occupée par la deuxième consonne de la racine:

(19)

fa $\sum_{\mathrm{C} V}^{1}$

(AspectP)

La troisième consonne de la racine, quant à elle, s'associe à une position $\mathrm{CV}$ sous AgreementP:

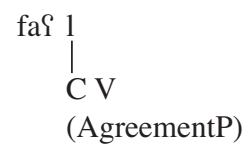

Ce qui donne en définitive, pour une forme I, le gabarit de base:

(20)

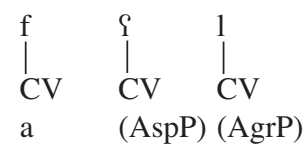


Ainsi, le gabarit tel qu'il est proposé par l'analyse morpho-phonologique de Guerssel \& Lowenstamm se construit au fur et à mesure que des projections syntaxiques sont ajoutées. Le gabarit sera perçu comme étant le résultat d'opérations diverses et non pas comme le lieu même de ces opérations.

\subsubsection{Le domaine $v P$}

La forme II Sallam ajoute une projection qui comporte un argument et un verbe du type «faire/rendre». Ce dernier n'ayant pas un sens bien défini ni un véritable contenu phonétique autonome, il ne sera pas de même nature que le verbe de base. En effet, la forme II Sallam contient un verbe construit à partir d'une racine $\sqrt{ } \mathrm{S} / \mathrm{m}$, qu'on appellera verbe lexical, et un autre, au sens de 'faire/ rendre', établi par une structure syntaxique, qu'on appellera verbe fonctionnel. Ce dernier est à rapprocher des verbes légers du français, à la différence près que ces derniers ont un contenu phonétique bien établi et peuvent dans d'autres structures fonctionner comme un vrai verbe lexical. $\mathrm{Ce}$ qui importe, c'est que les verbes ajoutés par une forme II ont la même fonction que les verbes légers et, par conséquent, nous pensons qu'ils ont la même structure.

De ce fait, la projection ajoutée par un verbe de forme II et qui apporte un argument et un verbe du type "faire/rendre" à la forme de base n'est autre que la projection vP. Nous proposons, donc, que la forme II Sallam, tout en disposant de la même structure qu'une forme I Salim (22a), à savoir une projection $\mathrm{VP}$ et une projection $\sqrt{\mathrm{P}}$, possède en plus une projection $\mathrm{vP}(22 \mathrm{~b})$ :

a.

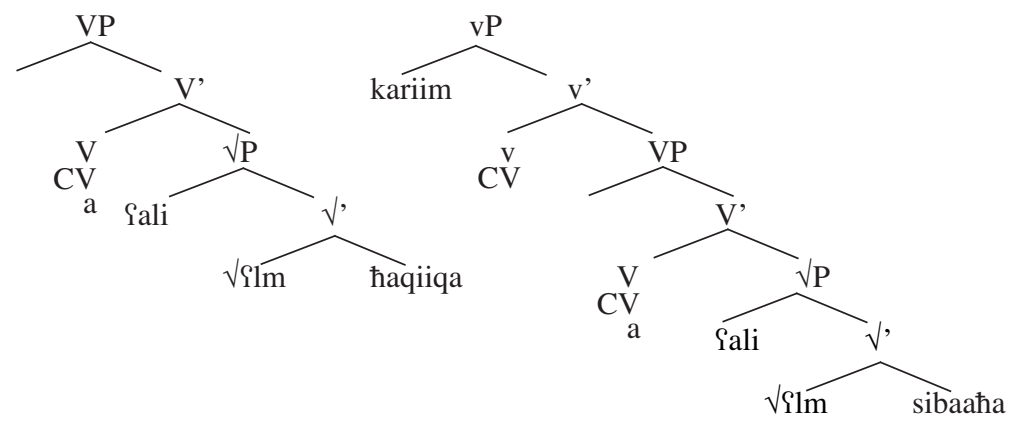

La projection $\mathrm{vP}$ procure une position tête qui permet d'ajouter un prédicat et une position Spécifieur qui permet d'ajouter un argument. Le verbe sous $\mathrm{v}$ n'ayant pas un vrai contenu phonétique et l'existence de cette position provoquant la gémination de la deuxième consonne de la racine, nous postulons que la position v contient une position CV. Ajoutons à cela qu'en arabe classique les têtes fonctionnelles sont souvent limitées à un CV 
et que plusieurs opérations morphologiques peuvent survenir au niveau et en considération d'un CV.

Ainsi, si le français réalise des verbes légers qui ont un contenu phonétique bien établi mais dont la notion sémantique est limitée, l'arabe classique réalise dans cette même position, une forme qui, en plus d'avoir un contenu sémantique limité, a un contenu phonologique minimum représenté sous forme d'une seule position CV.

\subsection{Processus de formation de la forme II causative}

Pour obtenir la forme II Gallam à partir de la structure en (22b), une série de mouvements est effectuée. Ces mouvements dépendront essentiellement de la structure qu'on a dégagée de l'étude de l'exemple avec la forme fallam: [Karim fait [Yali sait]]. On tiendra compte du fait qu'on a d'abord [Sali sait], à quoi on ajoute [Karim fait]. De la sorte, le premier mouvement consiste à déplacer la racine dans la position $\mathrm{V}$, de la même manière que dans la forme $\mathrm{I}$ Salim:

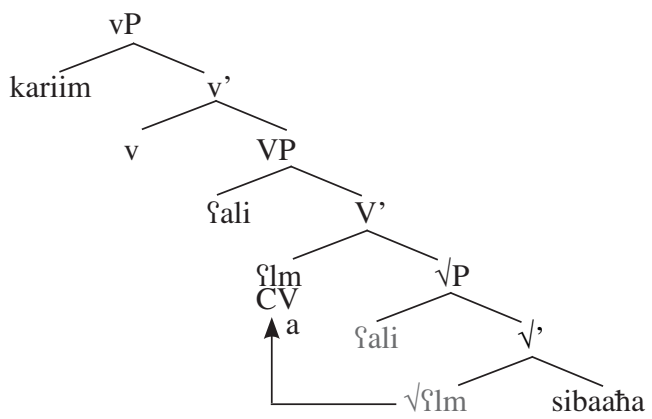

La racine se trouve ainsi associée à la position $\mathrm{C}$ du $\mathrm{CV}$ contenu dans $\mathrm{V}$. L'argument "Sali", initialement en position Spécifieur de $\sqrt{ } \mathrm{P}$, se retrouve dans la position Spécifieur de VP. Maintenant que VP est dominé par vP, c'est l'argument en position Spécifieur de vP qui acquerra le cas nominatif et aura la fonction Sujet. L'argument qui se trouve en position Spécifieur de VP aura le cas accusatif et la fonction Objet, ce qui explique la montée de V en v. Ajouté à cela que c'est le verbe sous v qui sera le verbe principal de la proposition, par conséquent, le morphème de la voix active contenu dans la position tête de VP doit se retrouver dans la position tête de vP. Ainsi, V monte dans v qui renferme également une position $\mathrm{CV}$ : 
(24)

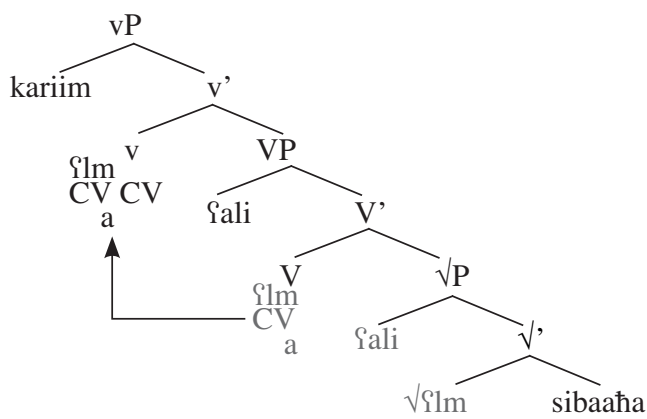

Suite à ce déplacement, $\mathrm{V}$ contenant une position $\mathrm{CV}$, dont la position vocalique est occupée par la voyelle "a" et dont la position consonantique est présentement occupée par la première consonne de la racine, se retrouve à gauche du CV sous v. La deuxième et la troisième consonne de la racine seront associées aux positions apportées respectivement par AspectP et AgreementP:

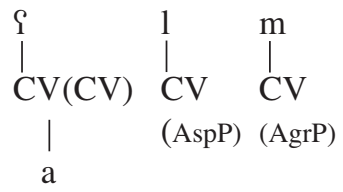

La position (CV) apportée par v se retrouve entre celle apportée par V et celle apportée par AspectP. N'ayant pas de contenu segmental propre qui lui est associée et n'étant pas en première position, auquel cas elle aurait pu être identifiée par une consonne épenthétique, cette position sera remplie par l'une des consonnes de la racine. Au regard de sa place, le choix se fera entre la première et la deuxième consonne de la racine. Cependant, la position $\mathrm{V}$ qui sépare la position $\mathrm{C}$ accueillant la première consonne de la racine et la position $\mathrm{C} d u(\mathrm{CV})$ en question n'est pas vide. Par conséquent, la première consonne de la racine ne pourra pas se propager en raison d'une prohibition indépendamment établie pour l'arabe classique ${ }^{3}$, et c'est la deuxième consonne de la racine qui occupera donc la position CV apporté par v.

Avec une seule consonne attachée à deux positions consonantiques on obtient une consonne géminée:

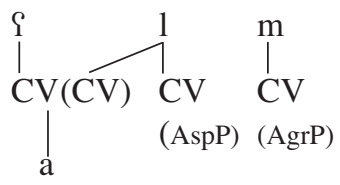

C'est ainsi que l'on obtient la forme II causative Sallam.

3. Dans le modèle $\mathrm{CVCV}$, une géminée est associée à deux positions $\mathrm{C}$ séparées pas une position $\mathrm{V}$ vide. 
Ainsi, la forme II faৎৎal renferme une structure du causatif qui apporte l'argument externe et un verbe sous-spécifié qui tiendra son sens "faire / rendre" de la structure syntaxique elle-même, mais également de la racine qui se prête à cette interprétation sémantique. Ce verbe n'a pas un contenu phonétique mais seulement une position CV, la même position dérivationnelle que l'on retrouve dans le gabarit proposé par Guerssel \& Lowenstamm (1990).

Maintenant que l'on a établi que la forme II faৎৎal est tout simplement obtenue avec une structure du causatif sans avoir à postuler l'existence d'autres projections ou mettre des étiquettes à la racine, reste à comprendre comment sont réalisées les autres formes II qui ne partagent avec le causatif ni le sens ni la structure.

Sachant qu'une forme II renferme une projection vP qui sélectionne une projection VP, sélectionnant elle-même une racine pouvant avoir diverses structures (i.e. une racine avec deux arguments dont l'un en position Spécifieur et le second en position Complément, une racine sélectionnant un seul argument en position Spécifieur ou encore une racine sélectionnant un seul argument en position Complément ou enfin une racine n'ayant aucun argument, en plus des différents rôles thématiques que peut jouer chacun de ses arguments et des traits sémantiques qui constituent chaque racine), on peut d'ores et déjà s'attendre à avoir autant de formes II que de structures différentes de la racine.

\section{La forme II faSSal intensive}

Le deuxième sens pour lequel la forme II faŞal est connue, après le sens causatif, est le sens intensif, sens qui lui est spécifique et qu'elle ne partage avec aucune autre forme (contrairement au causatif que la forme II partage avec la forme IV). Cette intensité peut se traduire soit par la violence de l'action (intensive) ou par la durée de l'action "temporally extensive", ou par le nombre des actants de l'action "numerically extensive" ou alors, la fréquence voire la répétition de l'action "iterative or frequentative" (Wright, $1896: 31$ ).

Soit les exemples suivants d'une forme II intensive:

(27) kassara Saliyy-un al-baab-a

a fracassé Sali-nominatif la porte-accusatif

'Pali a fracassé la porte'

(28) qațaia al-walad-u al-waraq-a

a découpé le garçon-nominatif le papier-accusatif

'le garçon a découpé le papier'

Avec les formes I correspondantes, on a ce qui suit:

(29) kasara @aliyy-un al-baab-a

a cassé Yali-nominatif la porte-accusatif 
'Sali a cassé la porte'

(30) qataia al-walad-u al-waraq-a

a coupé le garçon-nominatif le papier-accusatif

'le garçon a coupé le papier'

Contrairement à la forme II véhiculant un sens causatif, la forme II intensive ne provoque aucun changement quant au nombre des arguments. En effet, la forme II, ici, réalise les mêmes arguments que la forme I correspondante et chacun d'eux conserve le même rôle thématique et la même fonction syntaxique. Si l'on prend les exemples en (27) et (29), on voit que « Sali» qui est Agent et Sujet à la forme I kasara reste Agent et Sujet à la forme II kassar, de même, «al-baab» qui est Patient et Objet à la forme I, reste Patient et Objet à la forme II. Toutefois, les formes II citées ci-dessus présentent un sens d'intensité qui les différencie des formes I correspondantes:

$\begin{array}{lll}\text { kasar } & \rightarrow & \text { kassar } \\ {[\text { X casse }]} & \rightarrow & \text { [X casse plusieurs fois }] \\ \text { qatå } & \rightarrow & \text { qattå } \\ \text { [X coupe }] & \rightarrow & \text { [X coupe en petits morceaux }]\end{array}$

Ce sens qu'ajoute la forme II intensive est comparable à celui qu'on aurait avec un adverbe tel que «intensément», «abondamment», etc., mais aussi à celui qu'on aurait dans le cas d'une réduplication, comme si kassar ('casser plusieurs fois') était en réalité «kasar kasar» ('casser casser').

\subsection{Structure de la forme II intensive}

Reprenons un exemple de la forme II intensive:

(33) kassara Saliyy-un al-baab-a

a fracassé Sali-nominatif la porte-accusatif

'Sali a fracassé la porte'

Sachant qu'à la forme I on a la proposition :

(34) kasara Saliyy-un al-baab-a

a cassé Sali-nominatif la porte accusatif

'Sali a cassé la porte'

Le passage d'une forme I à une forme II change la forme verbale elle-même mais n'affecte pas le nombre d'arguments.

Partant du principe que la structure de la forme II prototype comporte une projection vP dont la tête apporte le verbe causatif et le Spécifieur l'argument supplémentaire, la forme II kassar ne semble pas répondre à ces critères : d'abord, elle ne contient pas un argument supplémentaire par rapport 
à la forme I. Ensuite, elle ne dénote pas le causatif ou une quelconque autre signification qui pourrait être rendue par un verbe léger (et qui serait tributaire du sens de la racine).

La forme II intensive kassar relève un défi parce qu'à la fois elle gémine la deuxième consonne de la racine et a exactement la même allure qu'une forme II causative, mais en même temps elle n'ajoute pas un argument externe, ni ne laisse apparaître un verbe léger. Cependant, nous présumons que les formes verbales dépendent d'une structure syntaxique bien déterminée qui est responsable de leur morphologie. De ce point de vue, si deux formes ont exactement la même allure, elles doivent avoir la même structure puisque c'est la structure syntaxique qui détermine la forme.

De la sorte, ou bien la forme II intensive présente un contre-exemple et montre que deux formes identiques morpho-phonologiquement peuvent avoir deux sources syntaxiques distinctes, ou bien ce qui distingue la forme II intensive de la forme II causative ne réside pas dans la partie de la structure qui engendre la gémination de la deuxième consonne de la racine et trouve son explication ailleurs. Avant de déterminer la structure de la forme II kassar, il convient d'exposer en premier la structure de la forme I kasar:

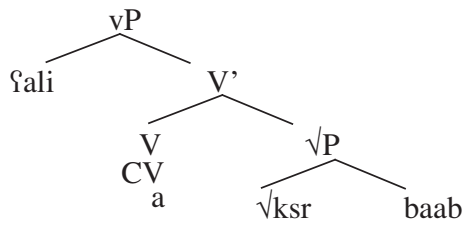

La racine $\sqrt{\mathrm{ksr}}$ fait partie des racines qui n'ont qu'un seul argument dont le rôle thématique est «patient» et dont la position est Complément. À la forme $\mathrm{I}$, l'argument «patient» de la racine occupant la position Complément de $\sqrt{\mathrm{P}}$ reste dans sa place et aura la fonction d'Objet direct. L'unique argument de la racine ne pouvant pas monter à la position Spécifieur de VP et être sujet - seul l'argument qui occupe la position Spécifieur de $\sqrt{ } \mathrm{P}$ peut le faire -, un argument est directement sélectionné par V. À la forme I kasar, cet argument (externe) est ajouté à la grille thématique de la racine, en VP.

Cette propriété de la racine $\sqrt{k s r}_{\mathrm{ks}}$ revêt une grande importance pour la compréhension de la forme II intensive car, tout d'abord, toutes les racines de ce type, lorsqu'elles font une forme II, sont intensives et, ensuite, parce que toutes les racines qui n'ont pas un argument en position Spécifieur se verront ajouter un argument par VP, et conjointement elles ne pourront plus avoir un deuxième argument externe.

Cette contrainte expliquerait pourquoi, dans le cas où la forme II intensive aurait la même structure que la forme II causative - à savoir une projection $\mathrm{vP}-$, celle-ci n'apportera pas un argument externe. En effet, même 
avec une projection $\mathrm{vP}$, si un argument externe est ajouté en VP et non pas apporté par la racine, il est impossible d'en ajouter un autre. C'est l'argument apporté par VP qui monte en position de Spécifieur de vP.

Toutefois, sil'on peut donner la raison, interne à la racine, qui expliquerait cette différence entre une forme II causative et une forme II intensive par rapport à l'ajout d'un argument externe, il reste à expliquer la deuxième caractéristique qui les distingue, à savoir pourquoi la forme intensive, si elle renferme bien une position $\mathrm{v}$, ne réalise pas un verbe léger du type «faire» ou «estimer» ou autre, en relation avec le sens et la structure de la racine.

Rappelons, tout d'abord, que la forme II kassar ne dénote pas exactement le même sens que la forme I kasar. Elle ajoute un trait sémantique par rapport à la forme de base qui se trouve être icil'intensif. Cet intensif, comme nous l'avons signalé, peut être rendu par une réduplication du verbe «kasar kasar». $\mathrm{Ce}$ qui supposerait l'existence d'un niveau verbal supplémentaire. Bien évidement, ici la réduplication n'est pas réalisée explicitement de la même manière que les verbes légers «faire» ou «estimer» que l'on fait ressortir dans les formes II causative et estimative.

À présumer que l'on a un niveau v, la question reste de savoir pourquoi on obtient un sens d'intensité au lieu d'un verbe léger du type «faire» qu'on retrouve dans la forme II causative. La réponse réside dans la structure de chacune de ces deux formes. Avec une forme II causative telle que Sallam, on a la structure: [karim v [ [ali Yalima as-sibaaha]] alors qu'avec la forme II intensive, on a [...v [Yali kasara al-ka?s]]. En l'absence d'un agent causateur, $\mathrm{v}$ ne peut pas dénoter l'événement «faire», voire «action».

La structure de la racine, obligeant à ajouter un argument au niveau de VP et empêchant de ce fait l'insertion d'un agent causateur au niveau de vP, élimine d'emblée le sens causatif mais aussi tout autre sens supposant l'existence d'un «agent» qui agit, de quelque manière que ce soit, sur un autre «agent» ou «thème». Par conséquent, $\mathrm{v}$ ne correspond pas à un quelconque événement et restera tel qu'il est en réalité, dépourvu de tout contenu sémantique. Le sens d'intensité sera acquis après le déplacement de la racine en $\mathrm{v}$ car avec un niveau verbal en plus, on obtient l'équivalent d'une réduplication de type «kasar kasar».

De la sorte, la forme II kassar renferme la même structure syntaxique que la forme II causative, à savoir une projection vP qui domine deux projections $\mathrm{VP}$ et $\sqrt{ } \mathrm{P}$ : 
(36)

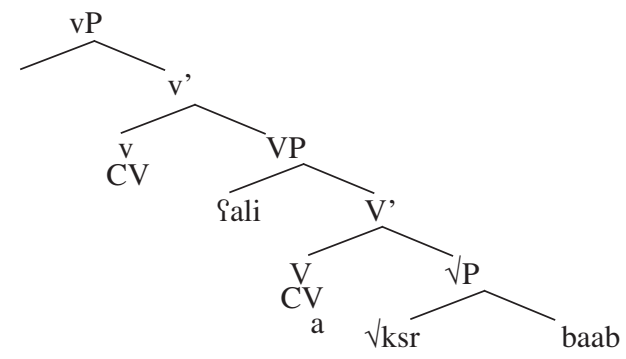

Lorsque la forme kasar est dominée par une projection vP, elle se trouve dotée d'une position v qui, contrairement à la forme II causative, ne sélectionne pas son propre argument. La projection vP contient, néanmoins, une position Spécifieur qui accueillera l'argument apporté par VP, lui permettant, par la suite, l'accès à la position Sujet. Ainsi, l'argument ajouté par VP, qui constitue le sujet et l'agent du verbe à la forme I kasar, monte à la forme II dans la position Spécifieur de vP et garde la même fonction syntaxique et le même rôle thématique. Il devient donc le sujet et l'agent de la forme II kassar.

\subsection{L'exécution de la forme II intensive}

Comme la forme II causative, la forme II intensive réalise une géminée au niveau de la deuxième consonne de la racine et la voyelle $a$ dans la même position que la forme I. La forme II intensive suivra le même processus de formation que la forme II causative.

Le fait que, dans la forme intensive, vP n'apporte pas un argument n'interfère en rien dans la construction d'une forme II. Celle-ci est obtenue lorsqu'on commence par mettre en place une forme I, condition remplie dans la forme II intensive puisque l'argument «agent» est ajouté en VP. La position de l'argument, à savoir s'il est dans VP et qu'il monte en vP ou s'il est sélectionné directement par v, n'intervient pas dans la formation même d'une forme II mais uniquement dans le sens que celle-ci va avoir.

Il suffit que la racine $\sqrt{ } \mathrm{ksr}$ monte en $\mathrm{V}$ et s'associe à la position $\mathrm{C}$ du $\mathrm{CV}$ contenu dans ce dernier (37a) et qu'ensuite $\mathrm{V}$ monte en v et se met à gauche de sa position CV (37b); 1'argument apporté par VP monte en position Spécifieur de vP: 
a.

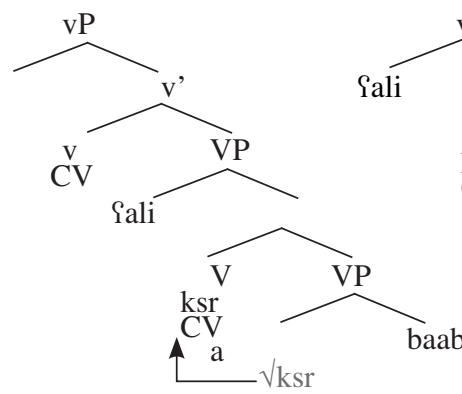

b.

Ayant procédé aux mêmes mouvements que la forme II causative, la forme II intensive revêt la même morphologie que celle-ci. La position CV apportée par v se retrouve à l'intérieur du gabarit suite à la montée de $\mathrm{V}$ et sera remplie par la deuxième consonne de la racine. Cette dernière étant déjà associée à la position $\mathrm{C}$ du $\mathrm{CV}$ de AspectP, elle se réalise géminée:

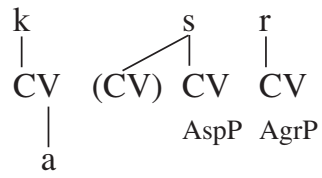

C'est ainsi que l'on obtient la forme II intensive kassar. Le fait qu'il $y$ ait un seul agent dans cette structure empêche la forme II d'avoir un sens causatif, et v n'ayant pas un sens bien défini, en l'absence d'un tiers agent, il devient simplement un niveau verbal de plus et rien d'autre. Le fait de faire déplacer un verbe de $\mathrm{V}$ à v lui attribue une sorte de second verbe, d'un point de vue structurel. De la sorte, un contenu sémantique répété dans deux positions verbales et ayant un même 'agent' produit un effet d'intensité.

Il apparaît ainsi qu'une forme II intensive n'est autre qu'une forme II causative ayant sélectionné une racine dont la structure argumentale ne comporte qu'un Complément ayant le rôle thématique de Patient.

\section{La forme II faŞal déclarative ou estimative}

On reconnaît à la forme II une valeur dite déclarative ou estimative (Wright 1896 : 31) dans le sens où l'on estime (voire déclare) la personne comme ayant la qualité dénotée par le verbe. Voici quelques exemples:

șaddaqa kariim-un Saliyy-an

a donné raison karim-nominatif (à) Sali-accusatif

'karim a donné raison à Yali' 
(40) kaddaba kariim-un Saliyy-an

a démenti karim-nominatif Yali-accusatif

'karim a démenti Sali'

Avec les formes I correspondantes, on a:

(41) șadaqa Saliyy-un

a raison Sali-nominatif

'Cali a raison'

(42) kadiba Saliyy-un

a menti Sali-nominatif

'Sali a menti'

Pareillement aux formes II causatives, les formes II dites déclaratives ou estimatives réalisent un argument en plus ainsi qu'un prédicat supplémentaire par rapport aux formes de base:

$$
\begin{array}{ll}
\text { șadaqa } & \rightarrow \quad \text { șaddaqa } \\
{[\mathrm{X} \text { a raison }]} & {[\mathrm{Y} \text { déclare } \mathrm{X} \text { a raison }]}
\end{array}
$$

(44) kadiba $\rightarrow$ kaddaba

[X ment] [Y déclare $\mathrm{X}$ ment]

L'argument apporté par la forme II est l'agent du prédicat «déclarer/ estimer». Celui réalisé à la forme I reste lié au prédicat «avoir raison/ mentir».

\subsection{Structure syntaxique de la forme II estimative}

Reconsidérons un des exemples cités ci-dessus :

(45) șaddaqa kariim-un Saliyy-an

a donné raison karim-nominatif Sali-accusatif

'karim a donné raison a Yali'

Sachant qu'à la forme I la proposition est la suivante:

(46) șadaqa Saliyy-un

a raison Sali-nominatif

'Sali a raison'

La forme II șaddaq ajoute un argument et un prédicat à la forme de base comme le fait la forme causative. Cependant, la forme II dont il est question ici n'ajoute pas un sens causatif à la forme I șadaqa mais ajoute un sens dit déclaratif/estimatif. Il n'est pas question, ici, de [faire + șadaqa] mais de [déclarer/estimer + șadaqa]. 
En dépit de cette distinction sémantique, le fait d'ajouter un argument et un verbe léger, et de réaliser une géminée de la même manière qu'une forme causative indique que la forme II estimative englobe également une projection $\mathrm{vP}$, et suit le même processus de formation qu'une forme II causative.

La structure de la forme II șaddaq se présente comme suit:

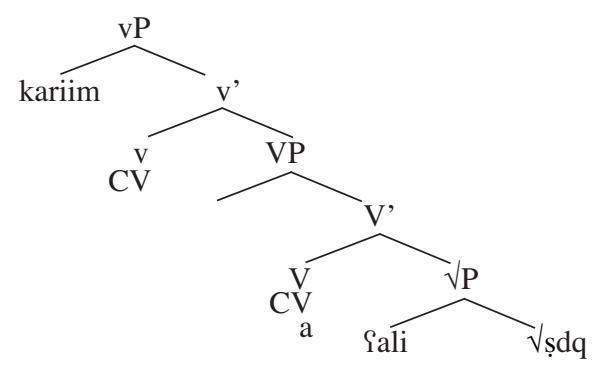

Si la forme II estimative șaddaq a la même structure que la forme II causative, mais exprime un sens distinct, l'explication ne peut résider que dans ce qui la différencie de la forme causative, à savoir la racine. La racine $\bigvee_{\text {șdq ne }}$ semble pas tolérer le trait [faire] et n'accepte pas une décomposition du type: [faire + avoir raison].

On sait que v n'a pas un contenu sémantique bien défini et représente uniquement un niveau verbal avec une position $\mathrm{CV}$. Le sens de ce prédicat est établi, voire résulte du sens de la racine: c'est la nature de la racine (si elle dénote une action ou un état) et sa structure (si elle a un seul argument ou deux), et le rôle thématique de ses arguments, qui donnent un sens à l'élément «verbe» sous v.

Ainsi, si dans une construction de type [karim v [ [ali Salima as-sibaaћa], le sens donné à v est «faire», dans la construction de type [karim v [Yali șadaq] le sens donné à v est «déclarer» ou «estimer». L'événement dénoté par la racine $\sqrt{ }$ șdq 'avoir raison' n'accepte pas un agent causateur: il ne s'agit pas d'un événement qui peut être provoqué par un tiers agent. De la sorte, une fois cette racine enchâssée dans une structure du causatif, le sens «action» obtenu sous $\mathrm{v}$ revêt le sens de «action psychique» au lieu de «action physique» et produit le sens «estimer».

\subsection{Processus de formation de la forme II estimative}

En ce qui concerne le processus de formation de la forme II estimative,

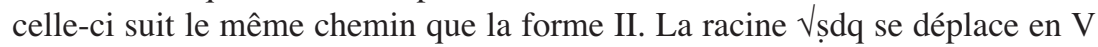
et s'associe à la position C du CV contenu dans ce dernier (48a); l'argument en position Spécifieur de $\sqrt{ } \mathrm{P}$ se retrouve dans la position Spécifieur de VP. Ensuite, V monte vers v et se met à sa gauche (48b): 
(48)

a.<smiles>[AlH2][AlH2][SiH3]</smiles>

(49) b.

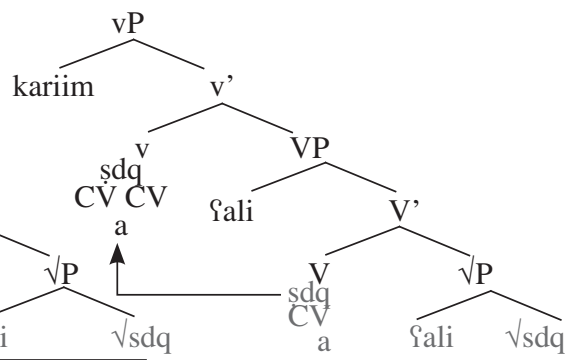

Il en résulte la même morphologie que la forme II causative :

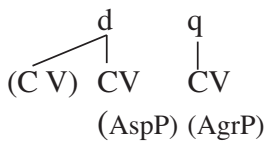

a

C'est ainsi que l'on obtient la forme II estimative șaddaq. Le fait que cette forme exprime un sens «estimatif/déclaratif» est dû à la valeur sémantique de la racine et ne change aucunement la structure syntaxique élaborée pour la forme II causative.

\section{La forme II faY̧al dénominative}

Les grammairiens attribuent à la forme II un emploi dénominatif et considèrent que cette forme sert également à former des verbes à partir de noms. Des exemples apparaissent ci-dessous :

(50) xayyama Saliyy-un

a campé Sali-nominatif

'Sali a campé'

(51) yarraba Saliyy-un

est parti vers l'ouest Sali-nominatif

'Sali est parti vers l'ouest'

Avec des formes nominales, on a ce qui suit:

(52) Raqaama Saliyy-un xaymat-an

a dressé Yali-nominatif une tente-accusatif

'Sali a dressé une tente'

(53) dahaba Saliyy-un ila al-yarb-i

est parti @ali-nominatif vers l'ouest-génitif

'Sali est parti vers l'ouest' 
La forme II xayyamqui signifie 'dresser une tente' ou 'camper' est considérée comme étant dérivée du nom xayma 'tente' et la forme II yarrab qui signifie 'partir à l'ouest' est considérée comme étant dérivée du nom yarb 'ouest'.

Ce que les grammairiens appellent une forme verbale dénominative, voire une forme verbale dérivée d'un nom, correspond dans notre analyse aux verbes formés à partir de racines qui renvoient à un objet du monde et qui ont la particularité, en principe, de n'avoir aucun argument.

Comme on peut le voir dans les exemples ci-dessus, la forme II dite dénominative manifeste un argument. De surcroit, elle ajoute un élément verbal qui semble être assez hétérogène et qui dépend étroitement du sens de la racine.

\subsection{Structure de la forme II dénominative}

Reprenons un des exemples de la forme II dite dénominative:

yarraba Saliyy-un

est parti vers l'Ouest Sali-nominatif

'Sali est parti vers l'ouest'

L'argument « Sali» qui occupe la position Sujet n'est pas apporté par la racine puisque cette dernière n'en a aucun, et la forme II yarrab semble contenir un verbe «partir» qui ne fait pas partie des traits sémantiques de la racine. Tout laisse donc supposer que la forme II dénominative répond parfaitement à la structure d'une forme II causative et englobe donc une projection vP qui lui attribue un verbe et un argument en plus.

Toutefois, comme on l'a déjà vu avec la forme II intensive, l'argument Sujet peut être ajouté en VP et ce lorsque la racine n'apporte pas un argument en position Spécifieur. Le cas ci-présent montre une racine qui n'apporte aucun argument. Par conséquent, l'argument réalisé par la forme II dénominative peut avoir été ajouté en VP au lieu d'être apporté par vP. Une façon de le vérifier est de voir si les formes dites dénominatives ont une forme I ou si elles n'existent que comme une forme II :

yarub-at af-Jams-u

disparaître à l'ouest le soleil-nominatif

'le soleil se couche à l'ouest'

On peut donc avoir un verbe de forme I yarub, à partir de la racine $\sqrt{ }_{\gamma} r b$, qui ne possède aucun argument. Ce qui indique que l'argument sujet est ajouté par VP. Si tel est le cas, alors dans la forme II yarrab, l'argument Sujet n'est pas apporté directement par vP mais est déjà sélectionné en VP. 
Pour comprendre ce qu'apporte un vP par rapport à un VP dans le cas de la racine nominale, examinons la différence entre yaruba et yarrab. La forme I yarub, indique 'être à l'ouest' alors que la forme II yarrab indique 'partir vers l'ouest'. Il semblerait ainsi qu'avec une racine nominale dont le sens réfère à un objet du monde, on obtient sous V un événement «être» et sous v un événement «faire», voire «action», qui peut prendre diverses significations selon la racine (ici, verbe de mouvement puisque la racine indique une destination).

Comme on l'a déjà observé dans le cas de la forme II intensive, le fait de ne pas apporter un argument n'exclut pas l'existence d'une structure causative, à savoir une projection vP. Par ailleurs, contrairement à la forme intensive, la forme II dénominative ajoute un verbe qui prend différentes significations selon la racine.

Ainsi, la forme II dénominative se distingue des autres formes II qu'on a vues jusqu'ici par le fait qu'elle est créée à partir d'une racine qui n'a pas d'arguments et qui donc ne projette pas. Cela ne l'empêche pas d'être dans une structure dominée par un vP. La structure de la forme II dénominative yarrab se présente comme suit:

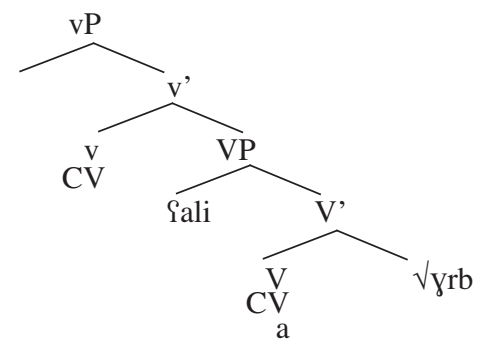

Le verbe produit par la forme II dénominative est en relation directe avec la racine et prend son sens dans celle-ci. Ici, comme la racine indique une direction 'ouest', le verbe qui en découle est un verbe de mouvement 'partir vers l'ouest'.

La forme II dénominative renferme plusieurs significations qu'on ne peut pas limiter de la même manière que pour la forme causative ou la forme estimative. Chaque forme dénominative a un sens différent qui dépend étroitement de la racine. La structure de la forme II ne fournit que le niveau verbal, et vu que la racine n'a pas d'arguments et n'exprime ni une action ni un état, le sens que la forme obtient sous v est tout simplement «action». Ensuite, ce verbe aura le sens que lui impose la racine.

\subsection{Processus de formation de la forme dénominative}

La forme II dénominative suit le même processus que les autres formes II. Que l'argument soit apporté par vP ou par VP n'interfère en rien 
dans la morphologie d'une forme II mais uniquement dans son sens puisque la position que ce dernier occupe ne concerne pas la forme elle-même.

La racine $\sqrt{\gamma}_{\gamma} \mathrm{rb}$, en position Complément de $\mathrm{V}$, s'associe à ce dernier et occupe la position C du CV qu'il renferme(57a). Ensuite V monte en v et se met à sa gauche et l'argument apporté par VP monte dans la position Spécifieur de vP(57b):

a.

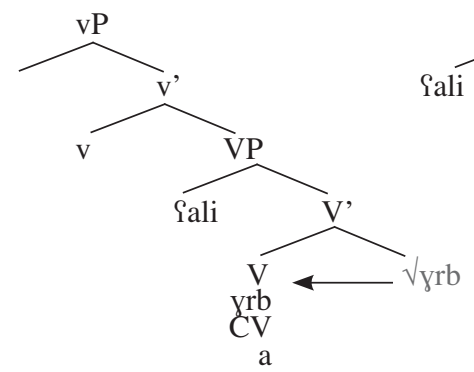

b.

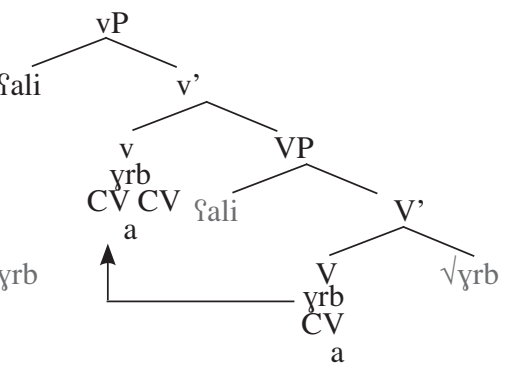

Ayant la même structure qu'une forme II causative et opérant les mêmes mouvements, la forme II dénominative revêt la même forme:

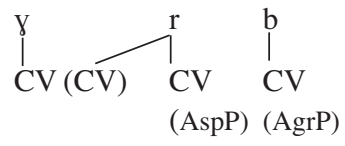

a

Il s'avère ainsi que la forme II dénominative est tout simplement une forme II qui renferme une racine sans argument. Cette propriété étant entendue, un argument est ajouté ipso facto au niveau de VP. Lorsque cette structure est dominée par vP, ce dernier ne peut pas apporter son propre argument. Le fait de ne pas avoir deux arguments dans cette structure déjoue le sens «causatif». Cependant, v procure à cette forme le sens «action»; sens qui sera par la suite précisé selon les traits sémantiques de la racine.

\section{Conclusion}

En dépit des différents emplois dans lesquels peut apparaître une forme II et des diverses significations qui lui sont attribuées, sa structure syntaxique reste unique. Toutes les formes II qui géminent la deuxième consonne de la racine ont une même structure, à savoir une projection vP qui s'ajoute à VP et à $\sqrt{ } \mathrm{P}$, et suivent le même procédé de formation. Ce qui change, d'une forme II à une autre, c'est la structure de la racine, laquelle déterminera si vP apporte un argument ou pas. Ce qui influe sur la sémantique de la forme II. 
Pour ce qui est de l'élément v, il forme une structure verbale supplémentaire et s'ajoute au verbe lexical constitué par la racine et par V. Il forge son sens en prenant en compte celui de la racine: il peut signifier soit 'faire', soit 'estimer' ou tout simplement 'action' et ce, dans le cas de la forme II dénominative. Plus encore, il peut se contenter de constituer juste une position en plus et ce, dans le cas de la forme II intensive. Ce verbe n'a pas un vrai contenu phonétique et se manifeste sous forme d'une position minimale [CV].

Ce qui produit la morphologie d'une forme II, à savoir la gémination de la deuxième consonne de la racine, c'est le fait que la racine monte d'abord en $\mathrm{V}$ et s'associe donc à la position consonantique du CV contenu dans ce dernier. Ensuite, $\mathrm{V}$ monte en $\mathrm{v}$ et se retrouve à gauche du $(\mathrm{CV})$ qu'il contient. Puisque la première consonne de la racine est séparée du (CV) de v par une voyelle "a", elle ne pourra pas se propager pour remplir cette position et c'est la deuxième consonne de la racine qui s'en charge, se réalisant ainsi géminée.

\section{RÉFÉRENCES}

Benmamoun, Elabbas (2000). The Feature Structure of Functional Categories. Oxford: Oxford University Press.

Benjaballah, Sabrina (2007). Constraints and markers in templates. In Picchi M. C. \& Pona A. (eds), Proceedings of the 32 Incontro di Grammatica Generativa: 21-34. Alessandria: Edizioni dell'Orso.

Сномsкy, Noam (1995). The Minimalist Program. Cambridge, Mass. : MIT Press.

Guerssel, Mohamed; Lowenstamm, Jean (1990). The Derivational Morphology of the Classical Arabic Verbal System. Ms. UQAM \& université Paris 7.

LAHROUCHI, Mohamed; SEgERAL, Philippe (2009). Morphologie gabaritique et apophonie dans un langage secret féminin en berbère tachelhit. Revue Canadienne de Linguistique 54/2: 291-316.

Lowenstamm, Jean (1996). CV as the only syllabe type. In Durand J. \& Laks B. (eds), Current Trends in Phonology, Models and Methods: 419-441. Salford, Manchester: ESRI.

Lowenstamm, Jean (2005). Deconstructing the Binyan. Afroasiatic Workshop. Leiden: Leiden University.

Lowenstamm, Jean (2003). À propos des gabarits. Recherches Linguistiques de Vincennes $32: 7-30$.

Marantz, Alec (1997). No escape from syntax: don't try morphological analysis in the privacy of your own lexicon. In Dimitriadis A. et al. (eds), UPenn Working papers in Linguistics 4/2: 201-225. Philadelphia, Pennsylvania: University of Pennsylvania.

RuCART, Pierre (2006). Morphologie gabaritique et interface phonosyntaxique - Aspects de la morphologie verbale de l'afar. Thèse de doctorat, université Paris 7.

SibaWAYH (1983 ed). Al-kitaab. Beyrouth: Saalam al kutub.

Wright, William (1896). A Grammar of the Arabic Language. Cambridge: Cambridge University Press. 


\begin{abstract}
Verbal measure II in Classical Arabic is characterized by geminated second consonant of the root. It vehicles different values: causative, intensive, estimative and denominative. Guerssel and Lowenstamm proposed a morphophonological analysis of this measure based on the existence of a derivational position (CV) in a template of the form : $\mathrm{CV}(\mathrm{CV}) \mathrm{CVCV}$, which allows the realization of the geminate. However, the different interpretations conveyed by this measure did not find a satisfying explanation within the frame of such analysis. This paper is an attempt at offering a syntactically-based analysis of measure II in Classical Arabic, that provides an explicit elucidation not only of the origin of the derivational $(\mathrm{CV})$ and the $\mathrm{CV}$ positions of the template, but also of the various semantic interpretations denoted by measure II. In the proposed analysis we demonstrate that the structure of verbal measure II includes VP, $\mathrm{vP}$ and a $\sqrt{\mathrm{P}}$ projection, just like French causative verb does. Furthermore, the root is not just considered as a sequence of three consonants, but as a domain that projects its own arguments. In this article, the value of the root existence is emphasized as well as the ability of syntax to offer solutions to problems long been considered morphophonological ones.
\end{abstract}

\title{
KeYwords
}

Syntax-phonology interface, Classical Arabic, verbal measure II, root structure, intensive, causative, template. 